\title{
Comparison of the discriminative stimulus effects of salvinorin A and its derivatives to U69,593 and $\mathrm{U} 50,488$ in rats
}

\author{
Lisa E. Baker • John J. Panos • Bryan A. Killinger • \\ Mary M. Peet • Lisa M. Bell • Larissa A. Haliw • \\ Sheryl L. Walker
}

Received: 19 May 2008 / Accepted: 28 December 2008 / Published online: 20 January 2009

(C) Springer-Verlag 2009

\begin{abstract}
Background and rationale Research interests regarding the psychopharmacology of salvinorin A have been motivated by the recreational use and widespread media focus on the hallucinogenic plant, Salvia divinorum. Additionally, kappa opioid (KOP) receptor ligands may have therapeutic potential in the treatment of some neuropsychiatric conditions, including drug dependence and mood disorders. Salvinorin A is a selective KOP agonist, but only a few studies have explored the discriminative stimulus effects of this compound.

Objective This study compared the discriminative stimulus effects of salvinorin $\mathrm{A}$ and two synthetic derivatives of salvinorin B to the KOP agonists, U69,593 and U50,488.

Materials and methods Sixteen male Sprague-Dawley rats trained to discriminate $\mathrm{U} 69,593(0.13 \mathrm{mg} / \mathrm{kg}$, s.c., $N=8)$ or U50,488 $(3.0 \mathrm{mg} / \mathrm{kg}$, i.p., $N=8)$ under a fixed-ratio 20 schedule of food reinforcement were administered substitution tests with salvinorin A $(0.125-3.0 \mathrm{mg} / \mathrm{kg}$, i.p.). The animals trained to discriminate U69,593 were also administered substitution tests with salvinorin B ethoxymethyl ether $(0.005-0.10 \mathrm{mg} / \mathrm{kg}$, i.p.) and salvinorin B methoxymethyl ether $(0.03-0.10 \mathrm{mg} / \mathrm{kg}$, i.p.). Another eight rats were trained to discriminate $2.0 \mathrm{mg} / \mathrm{kg}$ salvinorin $\mathrm{A}$ and tested with U69,593 (0.04-0.32 mg/kg) and U50,488 (0.4-3.2 mg/kg).

Results Salvinorin A and both synthetic derivatives of salvinorin B substituted completely for U69,593. Additionally, cross-generalization was observed between salvinorin $\mathrm{A}$ and both KOP agonists.
\end{abstract}

L. E. Baker $(\bowtie) \cdot$ J. J. Panos $\cdot$ B. A. Killinger $\cdot$ M. M. Peet $\cdot$

L. M. Bell $\cdot$ L. A. Haliw $\cdot$ S. L. Walker

Department of Psychology, Western Michigan University,

Kalamazoo, MI 49008, USA

e-mail: Lisa.Baker@wmich.edu
Conclusion These findings support previous reports indicating that the discriminative stimulus effects of salvinorin A are mediated by kappa receptors. Future studies may assist in the development and screening of salvinorin A analogs for potential pharmacotherapy.

Keywords Salvia divinorum · Salvinorin A . Kappa receptors $\cdot \mathrm{U} 69,593 \cdot \mathrm{U} 50,488 \cdot$ Salvinorin B EOM . Salvinorin B MOM $\cdot$ Drug discrimination $\cdot$ Rats

Salvia divinorum is a plant indigenous to Mexico that has been used in shamanistic rituals by the Mazatec people of Oaxaca for several centuries (Wasson 1962; Váldes et al. 1984, 1993). More recently, recreational use of this plant and its extracts has gained considerable popularity and widespread media attention in the United States and Europe. Indeed, recreational use of this substance is currently a popular topic of You Tube videos. Despite the growing popularity of this substance, its current legal status in the U.S. is unscheduled, which means growing, buying, and selling the plant are not regulated by the federal government. S. divinorum leaves and highly potent fortified extracts of this plant can be purchased legally over the Internet (Drug Enforcement Administration 2008; Prisinzano 2005a). The rising popularity of its recreational use has led to legal action in several of the U.S. states to ban the sale and use of $S$. divinorum and its extracts (Siebert 2007) and the Drug Enforcement Administration has placed it on the list of drugs of concern (Drug Enforcement Administration 2008).

Salvinorin A, the active constituent of $S$. divinorum, is a non-nitrogenous neoclerodane diterpene (Ortega et al. 1982; Váldes 1994). Currently the most potent naturally occurring hallucinogen (Váldes et al. 1984; Siebert 1994), salvinorin A is structurally distinct from all other known 
psychoactive compounds. Salvinorin A induces an intense and short-lived hallucinogenic experience reported to be qualitatively distinct from that induced by the classic hallucinogens, lysergic acid diethylamide and mescaline (Siebert 1994). Several studies have demonstrated that salvinorin A is a highly selective and potent kappa opioid (KOP) receptor agonist (Roth et al. 2002; Sheffler and Roth 2003; Chavkin et al. 2004; Yan and Roth 2004). Chavkin et al. (2004) examined the agonist activity at KOP receptors expressed in Xenopus oocytes with salvinorin A, U50,488, U69,593, and dynorphin A. Activity was determined by measuring potassium conductance through $G$ protein-gated $\mathrm{K}+$ channels. Results indicated that salvinorin $\mathrm{A}$ was significantly more efficacious than either U50,488 or U69,593 in this assay and comparable in efficacy to dynorphin $\mathrm{A}$, the endogenous ligand for KOP receptors.

In addition to clinical and scientific interests in characterizing the abuse liability of salvinorin $\mathrm{A}$, the unique pharmacological profile of this compound may lead to exciting prospects in the development of pharmacotherapeutics for neuropsychiatric disorders, including drug dependence (Prisinzano 2005b; Shippenberg et al. 2001, 2007). Thus, recent research efforts have focused on characterizing the behavioral effects of this compound in preclinical screening assays. There is now a considerable body of experimental research regarding the neurobehavioral effects of salvinorin A in a variety of animal species, including zebra fish, rodents, and rhesus monkeys. Many of the existing studies have compared the behavioral effects of salvinorin A with the synthetic KOP receptor agonist, U69,593, and reported substantial similarities between these compounds with regards to their effects on locomotor activity and motor coordination, sedative effects, and antinociceptive properties. For example, salvinorin A produced sedation and decreased motor coordination of mice in an inverted screen task (Fantegrossi et al. 2005), increased immobility and decreased swimming behaviors of rats in a forced swim test (Carlezon et al. 2006), and produced antinociceptive effects in mice in tail flick and hot plate tests of nociception (John et al. 2006; McCurdy et al. 2006).

Despite the recent surge in behavioral studies of salvinorin A, there is still insufficient scientific knowledge regarding the abuse liability of salvinorin $\mathrm{A}$ at the present time. In view of anecdotal reports and survey studies indicating that this substance tends to produce dysphoria (Gonzalez et al. 2006), the abuse liability of $S$. divinorum and its extracts may be relatively low compared to other recreational drugs. However, given the paucity of controlled scientific research, this conclusion may be a bit premature. A few investigators have recently examined salvinorin A in animal models of drug-induced reward, including conditioned place preference and intracerebroventricular (i.c.v.) self-administration (Zhang et al. 2005;
Braida et al. 2007; Braida et al. 2008). The results of these studies suggest that high doses of salvinorin A have aversive effects, although low doses may exert rewarding effects. Interestingly, Braida et al. (2008) reported that the reinforcing effects of low salvinorin $\mathrm{A}$ doses were attenuated by the CB1 antagonist rimonabant $(1 \mathrm{mg} / \mathrm{kg})$ as well as the KOP antagonist, nor-BNI $(10 \mathrm{mg} / \mathrm{kg})$. Using in vivo microdialysis techniques, Braida et al. (2008) also observed that a low dose of salvinorin A $(40 \mu \mathrm{g} / \mathrm{kg})$ increased extracellular dopamine levels by approximately $150 \%$ in the NAc shell. This finding is in contrast to previous findings that higher doses of salvinorin A decreased NAc DA levels (Zhang et al. 2005).

Further research is required to more fully characterize the psychopharmacology of salvinorin A. Drug discrimination procedures are a well-established animal model for classifying novel compounds with putative psychoactive actions because they allow for a comparison between novel substances and known psychoactive drugs (Schuster and Johanson 1988; Balster 1991). Salvinorin A has not yet been evaluated extensively in drug discrimination procedures, although a few studies have tested salvinorin $\mathrm{A}$ in rhesus monkeys (Butelman et al. 2004) or rats (Willmore-Fordham et al. 2007) trained to discriminate the kappa agonist U69,593 or monkeys trained to discriminate the serotonergic hallucinogen 2,5-dimethoxy-4-methylamphetamine (DOM; Li et al. 2008). Butelman et al. (2004) demonstrated stimulus generalization to salvinorin $\mathrm{A}$ in three rhesus monkeys trained to discriminate the kappa agonist, U69,593. Moreover, salvinorin A and U69,593 produced similar dosedependent and time-dependent functions, indicating similar stimulus properties of these two drugs. In contrast, the $\mathrm{N}$ methyl-D-aspartic acid receptor antagonist, ketamine, failed to substitute for U69,593, ruling out the possibility that animals might respond similarly to a hallucinogen with different neuropharmacological actions. The kappa receptor antagonists, quadazocine and 5'-guanidinonaltrindole (GNTI), blocked the substitution of salvinorin A, although GNTI effectively blocked salvinorin A substitution in only two of the three subjects tested.

In a more recent study, Willmore-Fordham et al. (2007) trained ten male Sprague-Dawley rats to discriminate $0.56 \mathrm{mg} / \mathrm{kg} \mathrm{U69,593} \mathrm{(i.p.,} 10 \mathrm{~min}$ ) and tested a range of salvinorin A doses $(1.0-3.0 \mathrm{mg} / \mathrm{kg}$ ) for substitution. At all doses tested, salvinorin A exhibited full substitution for U69,593 and these effects were blocked by i.c.v. administration of the kappa receptor antagonist, nor-BNI (4.5 nM), administered $60 \mathrm{~min}$ prior to salvinorin $\mathrm{A}$. These findings are consistent with those of Butelman et al. (2004). Together, these findings suggest that the discriminative stimulus effects of salvinorin A are mediated by kappa receptors. Only one published study to date has tested salvinorin $\mathrm{A}$ in animals trained to discriminate another 
hallucinogen. Li et al. (2008) recently reported that salvinorin A did not substitute for the discriminative stimulus effects of the serotonergic hallucinogen DOM in rhesus monkeys.

To date, there are no published reports of animals trained to discriminate salvinorin A. This may be related to the limited solubility of salvinorin A. In preliminary studies employing a vehicle comprised of ethanol/Tween $80 /$ sterile water (1:1:8 proportion, by volume), we experienced difficulties maintaining adequate stimulus control with salvinorin $\mathrm{A}$ in rats. Therefore, the current study used a different vehicle, $75 \%$ dimethyl sulfoxide (DMSO) in sterile water and successfully established discrimination with $2.0 \mathrm{mg} / \mathrm{kg}$ salvinorin A. The main objective of the present study was to determine whether stimulus generalization between salvinorin A and other kappa receptor agonists is symmetrical. In the first two experiments, salvinorin A was tested for substitution in animals trained to discriminate either U69,593 $(0.13 \mathrm{mg} / \mathrm{kg})$ or U50,488 $(3.0 \mathrm{mg} / \mathrm{kg})$. In addition, two synthetic derivatives of salvinorin B, the ethoxymethyl ether (salvinorin B EOM) and the methoxymethyl ether (salvinorin B MOM) were tested for substitution in the animals trained to discriminate U69,593. The chemical structures of these test compounds are depicted in Fig. 1. The third experiment represents the first study to establish discrimination with salvinorin A $(2.0 \mathrm{mg} / \mathrm{kg})$ and assess the effects of other kappa agonists for substitution.

\section{Materials and methods}

Subjects

Twenty-four male Sasco Sprague-Dawley rats (Charles River, Portage, MI, USA) were individually housed in
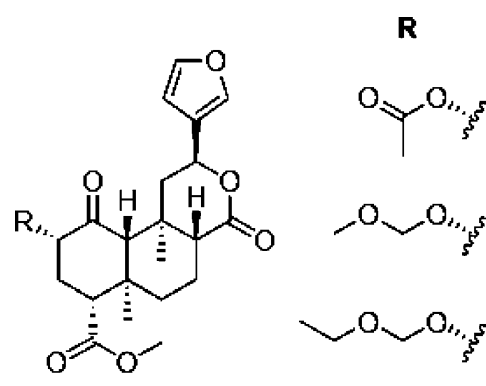

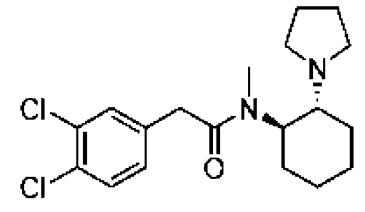

U50,488 salvinorin A

$\mathbf{R}$

salvinorin B MOM ether

salvinorin B EOM ether

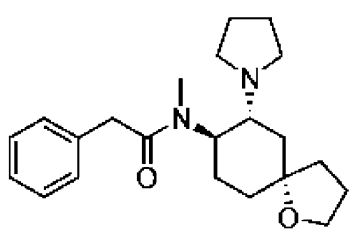

U69,593
Fig. 1 Structures of salvinorin A, salvinorin B MOM, and salvinorin B EOM in comparison to U50,488 and U69,593 polycarbonate cages with corn cob bedding in a colony maintained with a 12-h light/dark cycle (lights on 0700 to $1900)$ and constant temperature $\left(20 \pm 2^{\circ} \mathrm{C}\right)$ and humidity $(50 \pm 5 \%)$. Water was freely available in the home cages and commercial rodent diet was restricted to maintain body weights at $80-85 \%$ of free-feeding levels, accounting for age-related growth. Animals were maintained according to the general principles of animal husbandry outlined by the National Research Council (1996), and the experimental protocol was approved by the Institutional Animal Care and Use Committee of Western Michigan University.

\section{Apparatus}

Behavioral training and test sessions were conducted in 16 standard operant conditioning chambers (Med-Associates, Georgia, VT, USA) equipped with three retractable levers on the front panel, a food pellet delivery mechanism located above the center lever, and a $28-\mathrm{V}$ house light located at the top of the rear panel. Experimental events and data collection were computer-controlled using MED-PC (version 4.0 for Windows) instrumentation and software. Lever pressing was reinforced with dustless precision food pellets $\left(45 \mathrm{mg}\right.$, product \# F0021, Bioserv ${ }^{\circledR}$, Frenchtown, NJ, USA).

\section{Drugs}

Salvinorin A was obtained from the National Institute on Drug Abuse (Bethesda, MD, USA) and McLean Hospital (Belmont, MA, USA). Salvinorin B EOM and MOM were obtained from McLean Hospital. Due to limited solubility of these compounds, they were initially dissolved in DMSO and then diluted with sterile water to $75 \%$ DMSO. This vehicle has been used in a previous study (Willmore-Fordham et al. 2007) and was well-tolerated by the animals in the current study. There were no obvious signs of tissue necrosis following repeated injections with this vehicle. U69,593 (National Institute on Drug Abuse, Bethesda, MD, USA) was initially dissolved in a few drops of lactic acid and then diluted in sterile water. U50,488 (National Institute on Drug Abuse, Bethesda, MD, USA) was dissolved in sterile water. Drug or vehicle injections were administered intraperitoneally (i.p.) or subcutaneously (s.c.) in a volume of $1 \mathrm{mg} / \mathrm{mL}$. All drug doses were determined based on the weight of the salt.

Preliminary training

Prior to lever-press training, subjects were exposed to two 60-min sessions in which food pellets were delivered under a fixed-time $60 \mathrm{~s}\left(\mathrm{FT} 60^{\prime \prime}\right)$ schedule to familiarize animals with the sound and location of food reinforcers. During 
these sessions, no levers were present in the chambers. Animals were then trained to press the center lever for food pellets on a fixed-ratio 1 (FR 1) schedule during a single 20-min training session. This was the only session in which the center lever was used. Once animals were trained to lever press, a series of four to six errorless training sessions were conducted in which only the left lever or the right lever was present. Thirty minutes before these training sessions, animals were injected with the training drug (see below) or its vehicle solution. For half the animals in each group of eight rats, responses on the left lever were reinforced following drug administration and responses on the right lever were reinforced following vehicle administration. Conditions were reversed for the remaining animals in each group. An equal number of errorless sessions were conducted with each training condition. During these sessions, responding was initially reinforced under an FR 1 schedule and the response requirement was gradually increased from an FR 1 up to an FR 20 depending on an individual animal's performance. The FR was programmed to increment by two after every fifth reinforcer earned. The number of reinforcers that could be earned during a training session was limited only by the schedule and by the duration of the session. Once animals were reliably responding under both drug and vehicle conditions, discrimination training sessions commenced.

\section{Discrimination training}

For discrimination training sessions, both left and right levers were always present. These sessions lasted $20 \mathrm{~min} /$ day and were conducted approximately the same time of day 6 to 7 days/week. Drug and vehicle training sessions were alternated to include at least three drug training sessions and at least three vehicle training sessions per week with no more than two consecutive sessions under the same stimulus conditions. A resetting FR schedule of reinforcement was in effect, requiring a fixed consecutive number of correct responses for reinforcer delivery. Incorrect responses reset the response counter. Similar to the errorless training sessions, responding was initially reinforced under an FR 1 schedule and the response requirement was incremented by two after every fifth reinforcer up to a final FR 20. Once animals were responding reliably under a resetting FR 20 schedule under both drug and vehicle conditions, this schedule remained in effect for the remainder of training. Discrimination accuracy was determined by calculating the percentage of correct lever presses prior to the first food pellet delivery during each training session. When discrimination performance was $80 \%$ or greater for at least eight out of ten consecutive training sessions, an animal was said to have met the discrimination criterion. Three different groups of eight rats were trained to discriminate one of the following compounds: U69,593 $(0.13 \mathrm{mg} / \mathrm{kg}), \mathrm{U} 50,488(3.0 \mathrm{mg} / \mathrm{kg})$, or salvinorin A $(2.0 \mathrm{mg} / \mathrm{kg})$. Specific details regarding the training and testing of each group are described below in three separate experiments.

Stimulus generalization tests

Once animals met the criterion for stimulus discrimination, test sessions were conducted to determine substitution with a range of doses of the training drug. Following determination of dose-response curves with each training drug, three to four doses of each test compound were tested for substitution to the training stimulus. For each compound tested, the order of test doses was randomized among animals in each group. For each test dose, approximately half the animals were tested following a drug training session and the other half were tested following a vehicle training session. Test sessions were conducted once or twice per week depending on the performance of individual animals. Animals received a minimum of one drug training session and one vehicle training session between tests. Tests were conducted only when discrimination performance during these training sessions was at least $80 \%$. Test sessions were conducted in a similar manner to training sessions with the exception that no reinforcers were delivered and the animal was removed from the operant conditioning chamber immediately following completion of 20 consecutive responses on either lever.

\section{Experiment 1}

Eight rats were trained to discriminate $0.13 \mathrm{mg} / \mathrm{kg}$ U69,593 (s.c., $30 \mathrm{~min}$ ) and a dose-response curve was generated with the training drug following the procedures described above. Subsequently, these animals were administered substition tests with salvinorin A $(0.125-1.0 \mathrm{mg} / \mathrm{kg}$, i.p., $30 \mathrm{~min})$ salvinorin B EOM $(0.005-0.10 \mathrm{mg} / \mathrm{kg}$, i.p., $30 \mathrm{~min})$, and salvinorin B MOM $(0.03-0.10 \mathrm{mg} / \mathrm{kg}$, i.p., $30 \mathrm{~min})$.

\section{Experiment 2}

Eight rats were trained to discriminate $3.0 \mathrm{mg} / \mathrm{kg}$ U50,488 (i.p., $30 \mathrm{~min}$ ) according to the procedures described above. Subsequently, dose-response tests were conducted with U50,488 $(0.375-3.0 \mathrm{mg} / \mathrm{kg})$ and salvinorin A $(0.25-$ $3.0 \mathrm{mg} / \mathrm{kg}$, i.p., $30 \mathrm{~min}$ ) in five of these animals.

\section{Experiment 3}

Eight rats were trained to discriminate salvinorin A. The initial training dose selected was $0.5 \mathrm{mg} / \mathrm{kg}$ (i.p., $15 \mathrm{~min}$ ). 
Only two of the eight animals met the discrimination criteria in less than 50 training sessions. After 58 training sessions, the training dose was increased to $1.0 \mathrm{mg} / \mathrm{kg}$ and the injection interval was increased to $30 \mathrm{~min}$, based on observations that this dose substituted in the animals trained to disriminate U69,593 in experiment 1 . There was no improvement in the discrimination after an additional 30 training sessions, so the training dose was subsequently increased to $2.0 \mathrm{mg} / \mathrm{kg}$. Stimulus control was established under these conditions in seven of the eight animals. Substitution tests were conducted with the following compounds: salvinorin A $(0.25-2.0 \mathrm{mg} / \mathrm{kg}, N=7)$, U69,593 (0.04-0.32 mg/kg, N=5-6), and U50,488 (0.4$3.2 \mathrm{mg} / \mathrm{kg}, N=5-6)$.

Data analyses

The mean $( \pm$ SEM $)$ number of sessions to criterion were calculated for each training drug. For each training drug and test compound, dose-response curves were plotted to depict the percentage of responses made on the drugappropriate lever and the response rate (number of responses per second) at each dose. Group means $( \pm$ SEM) were calculated and plotted in the dose-response curves. The test data from animals that completed all doses of a particular test compound were also analyzed statistically using a repeated-measures analysis of variance followed by Dunnett's multiple comparison tests to determine if individual doses were significantly different from a vehicle control. Complete stimulus generalization at a particular dose of a test compound was defined as a group mean of $80 \%$ or greater drug-appropriate responding. If a test dose produced drug-appropriate responding that was less than $80 \%$ but significantly different from the amount of drugappropriate responding during vehicle tests, it was considered to produce partial substitution. Statistical analyses were conducted and graphs were created using Prism GraphPad (version 4.0) software (San Diego, CA, USA).

\section{Results}

\section{Experiment 1}

Rats trained to discriminate $0.13 \mathrm{mg} / \mathrm{kg} \mathrm{U} 69,593$ met the discrimination criterion within an average of $69( \pm 11.4$, SEM) training sessions (range 36-136). Figure 2 depicts the dose-response curves obtained from the results of substitution tests with U69,593, salvinorin A, salvinorin B $\mathrm{EOM}$, and salvinorin B MOM in these animals. There was a statistically significant effect of U69,593 dose on the percentage of drug-appropriate responses $\left(F_{4,39}=12.57, p<\right.$ 0.0001 ) and all except the lowest dose of U69,593 were

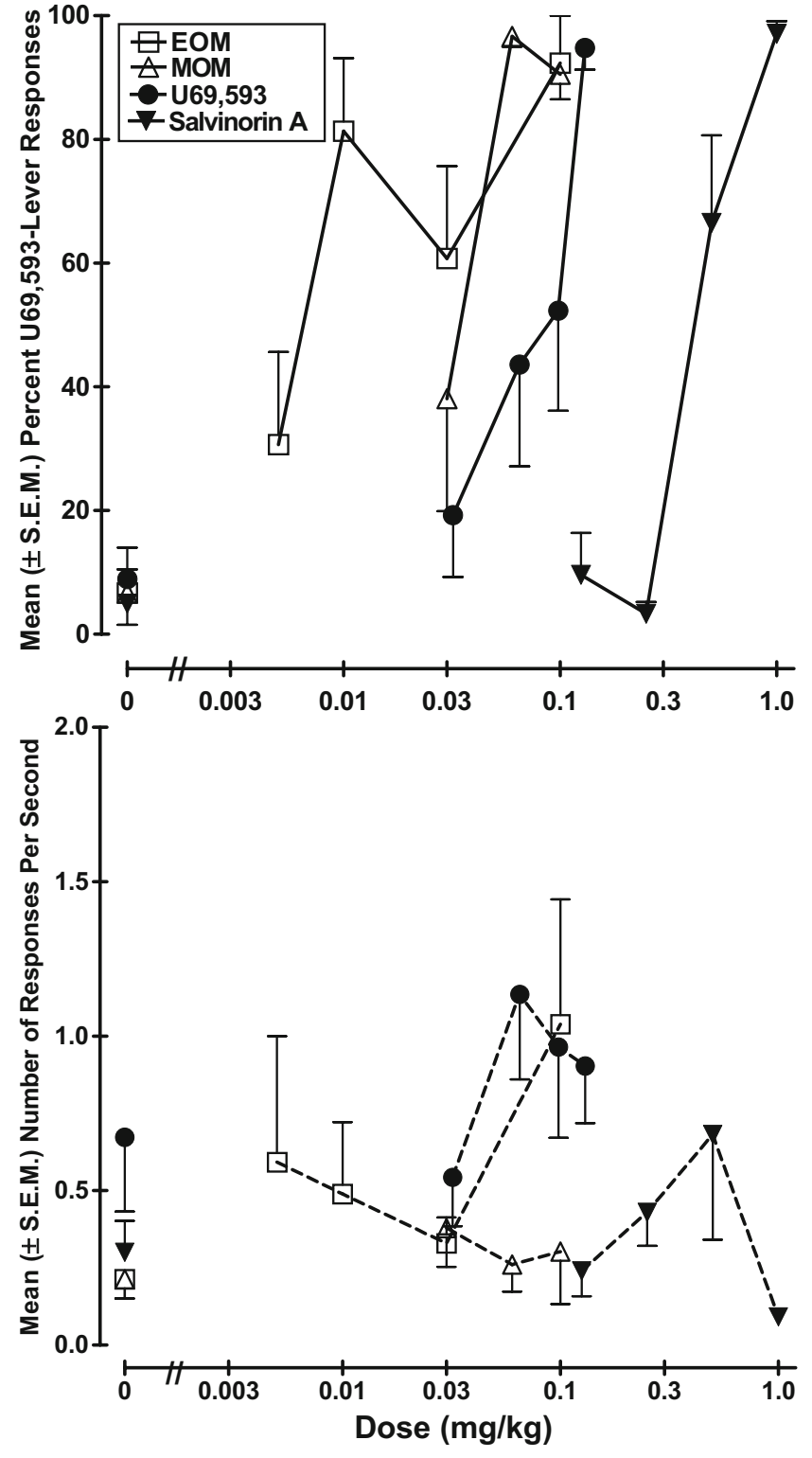

Fig. 2 Dose-response functions for U69,593 $(N=8)$, salvinorin A $(N=$ $8)$, salvinorin B EOM $(N=7-8)$, and salvinorin B MOM $(N=8)$ in rats trained to discriminate U69,593 (0.13 mg/kg, s.c., $30 \mathrm{~min})$. Discrimination accuracy data are displayed in the top graph and response rates are displayed in the bottom graph. Data points represent group means $( \pm$ SEM)

significantly different from vehicle $(0.065$ and $0.098 \mathrm{mg} / \mathrm{kg}$, $p<0.05$ and $0.13 \mathrm{mg} / \mathrm{kg}, p<0.01$ compared to vehicle). U69,593 increased response rate relative to vehicle, but this effect was not statistically significant.

As expected, salvinorin A produced dose-dependent increases in U69,593-appropriate responding and substituted fully for this training drug at $1.0 \mathrm{mg} / \mathrm{kg}$. Salvinorin A significantly increased the percentage of U69,593-appropriate responses $\left(F_{4,39}=34.90, p<0.0001\right)$ and the 0.5 and $1.0 \mathrm{mg} / \mathrm{kg}$ doses were significantly different from vehicle 
$(p<0.01)$. Effects of salvinorin A on response rate were not statistically significant. Salvinorin B EOM and salvinorin B MOM produced complete substitution for U69,593 at considerably lower doses compared to salvinorin A. Statistical tests showed a significant effect of salvinorin B EOM on drug-appropriate responses $\left(F_{4,19}=13.73, p<\right.$ 0.001 ) with three doses $(0.01,0.03$, and $0.10 \mathrm{mg} / \mathrm{kg}$ ) significantly different from vehicle $(p<0.01)$. Full substitution was obtained with both 0.01 and $0.10 \mathrm{mg} / \mathrm{kg}$, but not $0.03 \mathrm{mg} / \mathrm{kg}$ salvinorin B EOM. The effects of salvinorin B MOM on drug-appropriate responding were also statistically significant $\left(F_{3,27}=15.16, p<0.0001\right)$; both 0.06 and $0.10 \mathrm{mg}$ were significantly different from vehicle $(p<$ 0.01 ) and produced full substitution for U69,593. Effects of these compounds on response rate were not significantly different from vehicle control rates.

\section{Experiment 2}

Rats trained to discriminate U50,488 met the criteria for discrimination specified above within an average of 38.8 ( \pm 4.4 , SEM) training sessions (range 27-65). Although all eight rats met these criteria, only five of the eight animals maintained reliable discrimination to complete all the stimulus generalization tests. Figure 3 depicts the doseresponse curves generated from stimulus generalization tests with the U50,488 and salvinorin A in these five rats. The effects of U50,488 doses on the percentage of druglever responses were statistically significant $\left(F_{4,24}=3.69\right.$, $p<0.05)$. Only the training dose was significantly different from vehicle control $(p<0.01)$. Salvinorin A produced dose-dependent increases in drug-appropriate responding and substituted fully for U50,488 at $3.0 \mathrm{mg} / \mathrm{kg}$. One animal did not respond at the highest dose of salvinorin $\mathrm{A}$, so the data for this animal were excluded from statistical analyses. The effects of salvinorin A on the percentage of U50,488appropriate responding were statistically significant $\left(F_{5,23}=\right.$ $4.12, p<0.05)$ and both $2.0 \mathrm{mg} / \mathrm{kg}(p<0.05)$ and $3.0 \mathrm{mg} / \mathrm{kg}$ $(p<0.01)$ produced drug-appropriate responding that was significantly different from vehicle. Response rates were slightly increased by U50,488 and slightly reduced by salvinorin A relative to vehicle control levels, although neither of these drug's effects on response rate were statistically significant.

\section{Experiment 3}

The initial training dose of salvinorin A $(0.5 \mathrm{mg} / \mathrm{kg}$, i.p., $15 \mathrm{~min}$ ) established stimulus control in two animals within 44 and 47 training sessions, respectively. As noted above, the training dose was increased to $1.0 \mathrm{mg} / \mathrm{kg}$ (i.p., $30 \mathrm{~min}$ ) after 58 training sessions and then to $2.0 \mathrm{mg} / \mathrm{kg}$ after another 30 training sessions. Seven of the eight rats met the
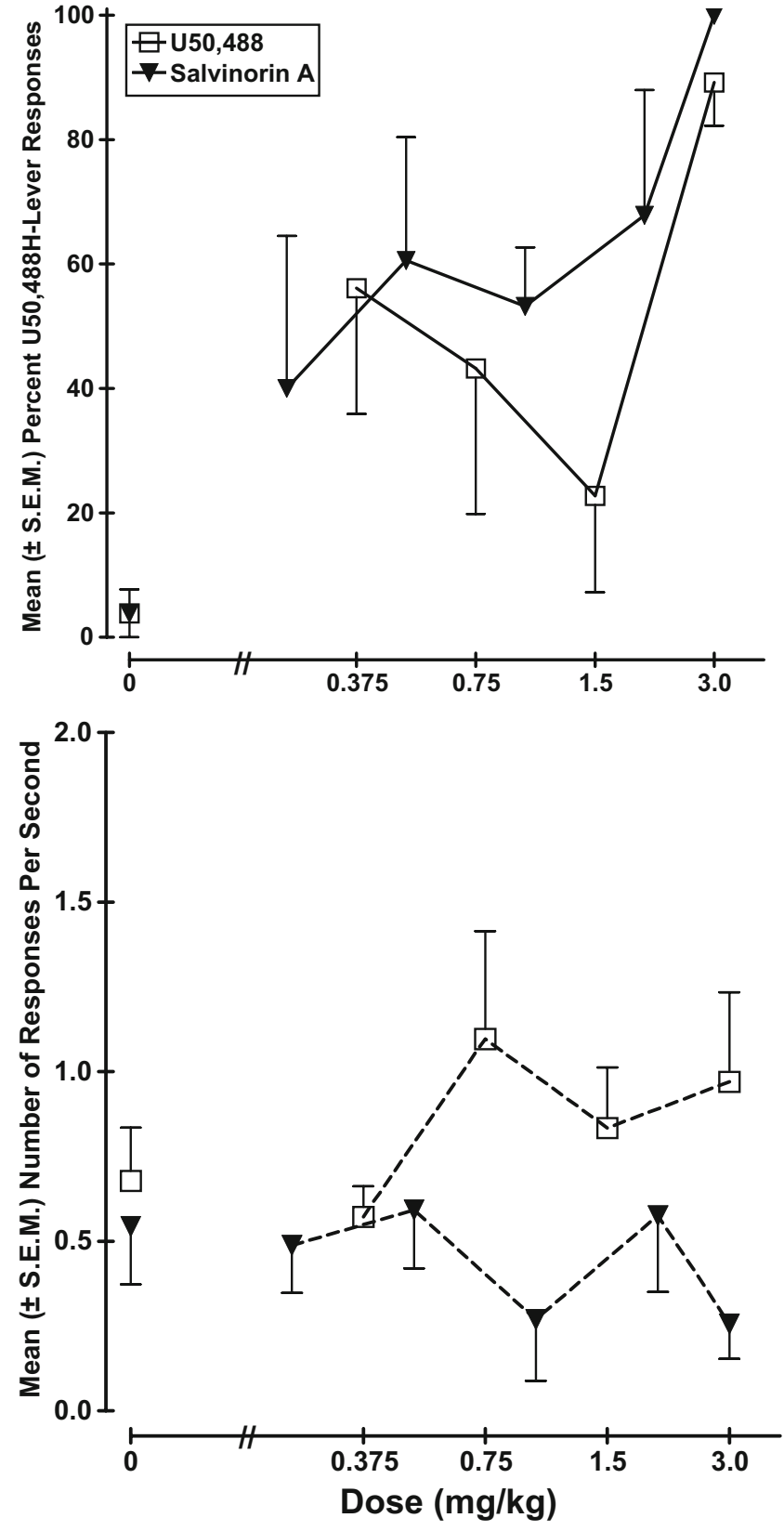

Fig. 3 Dose-response functions for U50,488 $(N=5)$ and salvinorin A $(N=5)$ in rats trained to discriminate U50,488 $(3.0 \mathrm{mg} / \mathrm{kg}$, i.p., $30 \mathrm{~min})$. See Fig. 1 for additional details

criteria for discrimination within an average of $19.7( \pm 7.2$, SEM) additional training sessions (range 10-62) after the dose was increased to $2.0 \mathrm{mg} / \mathrm{kg}$. Figure 4 depicts the doseresponse curves generated from stimulus generalization tests with salvinorin A, U69,593, and U50,488 in these animals. Salvinorin A produced dose-dependent increases in discrimination accuracy and both 1.0 and $2.0 \mathrm{mg} / \mathrm{kg}$ produced complete stimulus generalization. Statistical analyses on the percentage of drug-appropriate responding revealed a significant effect of salvinorin $\mathrm{A}$ dose $\left(F_{4,29}=\right.$ 

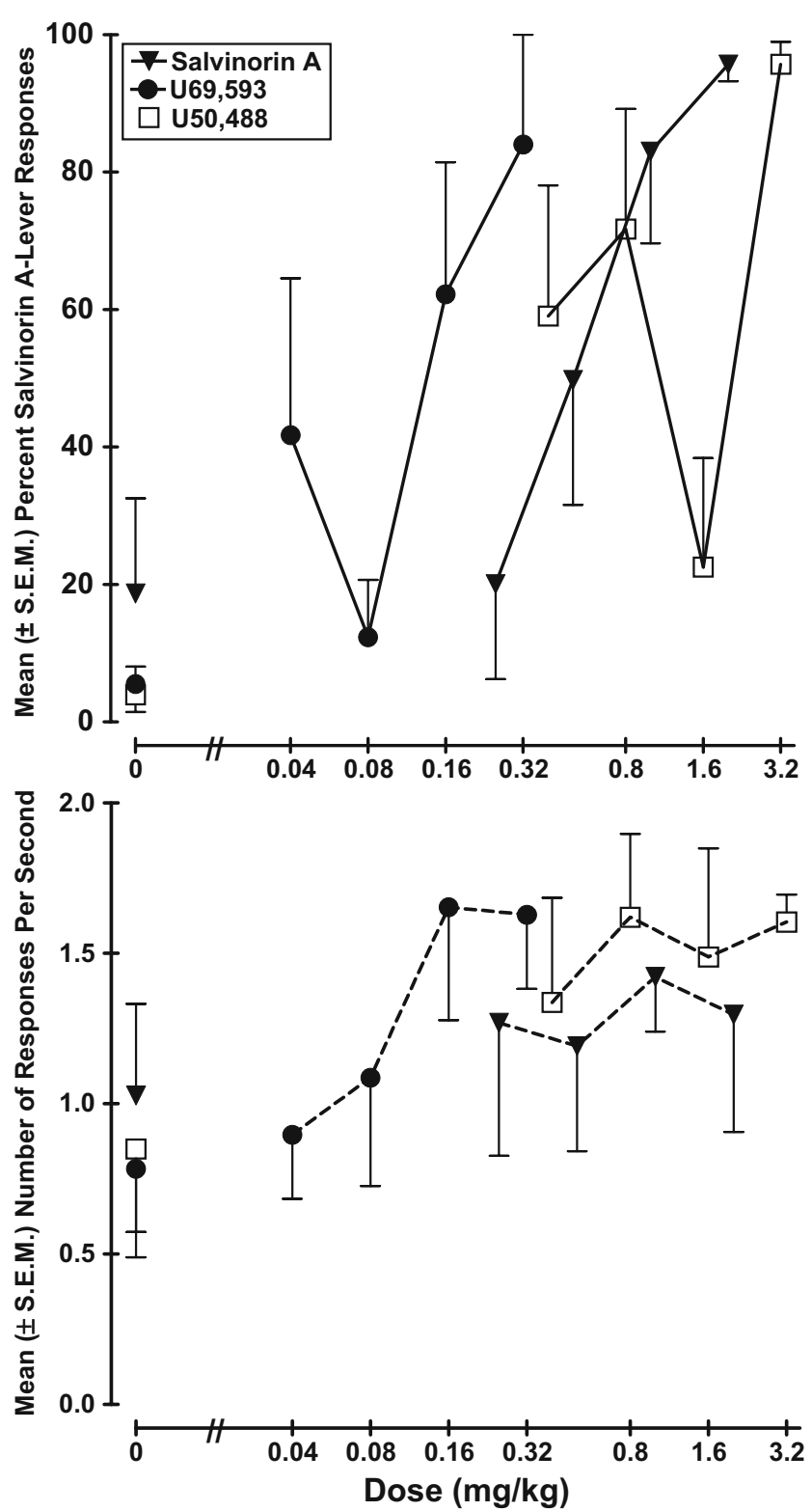

Fig. 4 Dose-response functions for salvinorin A $(N=7)$, U69,593 $(N=$ 5-6), and U50,488 $(N=5-6)$ in rats trained to discriminate salvinorin A $(2.0 \mathrm{mg} / \mathrm{kg}$, i.p., $30 \mathrm{~min})$. See Fig. 1 for additional details

7.44, $p<0.001)$. Response rates were not significantly different among different doses of salvinorin A.

Both U69,593 $(0.32 \mathrm{mg} / \mathrm{kg})$ and U50,488 $(3.2 \mathrm{mg} / \mathrm{kg})$ produced complete stimulus generalization to salvinorin A. The effects of U69,593 $\left(F_{4,24}=5.22, p<0.01\right)$ and $\mathrm{U} 50,488$ $\left(F_{4,24}=5.35, p<0.01\right)$ on salvinorin A-appropriate responses were statistically significant; $0.32 \mathrm{mg} / \mathrm{kg}$ U69,593 $(p<0.01)$ and two doses of U50,488 $(0.8 \mathrm{mg} / \mathrm{kg}$, $p<0.05$ and $0.32 \mathrm{mg} / \mathrm{kg}, \quad p<0.01$ ) were significantly different from vehicle control. The large error bars at some doses are indicative of disparate results among subjects. For example, U50,488 $0.8 \mathrm{mg} / \mathrm{kg}$ produced $95 \%$ to $100 \%$ salvinorin A-responding in four of the six animals tested, and only $5 \%$ and $31 \%$ salvinorin A-responding in the other two animals. Complete substitution was observed with $3.2 \mathrm{mg} / \mathrm{kg} \mathrm{U} 50,488$ in all five animals tested at this dose. Similarly, lower doses of U69,593 produced complete substitution in some animals and vehicle-appropriate responding in other animals. The $0.32-\mathrm{mg} / \mathrm{kg}$ dose of U69,593 produced $100 \%$ salvinorin A-responding in five of the six animals tested and vehicle-appropriate responding in one animal. Interestingly, both U69,593 and U50,488 increased response rates in a dose-dependent manner, but these effects were not significantly different than vehicle control rates of responding.

\section{Discussion}

Previous studies have demonstrated that salvinorin A substitutes in monkeys (Butelman et al. 2004) or rats (Willmore-Fordham et al. 2007) trained to discriminate the kappa agonist, U69,593, and that these effects are attenuated by KOP antagonists. The present results are consistent with these reports and extend these findings to a lower dose of the U69,593 and to another kappa agonist, U50,488. Willmore-Fordham et al. (2007) trained rats to discriminate a considerably higher dose of U69,593 $(0.56 \mathrm{mg} / \mathrm{kg}$, i.p., $10 \mathrm{~min}$ ) than the one employed in the current study $(0.13 \mathrm{mg} / \mathrm{kg}$, s.c., $30 \mathrm{~min})$. In addition, all three doses of salvinorin A that they tested $(1.0,1.9$, and $3.0 \mathrm{mg} / \mathrm{kg}$ ) produced full substitution for U69,593 without significantly reducing response rates. The current study tested a lower range of salvinorin A doses $(0.125-1.0 \mathrm{mg} / \mathrm{kg})$ in order to generate a complete dose-response curve. Only the $1.0-\mathrm{mg} /$ $\mathrm{kg}$ dose produced complete substitution in animals trained to discriminate $0.13 \mathrm{mg} / \mathrm{kg} \mathrm{U69,593}$. Although these doses did not significantly reduce response rates in the current study, a higher dose of salvinorin A $(2.0 \mathrm{mg} / \mathrm{kg})$ produced sedative effects and impaired responding in animals trained to discriminate $0.13 \mathrm{mg} / \mathrm{kg} \mathrm{U} 69,593$. The $2.0-\mathrm{mg} / \mathrm{kg}$ salvinorin A test data are not graphed because none of the animals administered this dose made enough responses to be included in the analyses. This was not the case with animals trained to discriminate $0.56 \mathrm{mg} / \mathrm{kg} \mathrm{U} 69,593$ in the Willmore-Fordham et al. (2007) study or in animals trained to discriminate $3.0 \mathrm{mg} / \mathrm{kg}$ U50,488 in the present study. The differences in the effects of salvinorin $\mathrm{A}$ on response rates in these studies are likely a result of exposure to different doses of the training compounds.

The current study also represents the first known demonstration that rats can be trained to discriminate salvinorin A and that both U69,593 and U50,488 substitute for this compound, showing evidence for symmetrical generalization between salvinorin A and other KOP 
agonists. A more complete characterization of the discriminative cue properties of salvinorin A will require tests of stimulus generalization between this substance and a variety of psychoactive compounds representative of different pharmacological classes. Such studies are an essential component to a thorough investigation of the abuse liability of salvinorin A.

It has been suggested that salvinorin A, by virtue of its potency, efficacy, and selectivity as a KOP receptor agonist, may serve as an important tool for discovery regarding the role of the dynorphin/kappa opioid (DYN/KOP) system in neurological diseases and neuroadaptation (Chavkin et al. 2004; Prisinzano 2005b; Shippenberg et al. 2001, 2007). Relatively few synthetic compounds have been developed that have a high selectivity for KOP receptors. Until recently, the most specific of these compounds were U69,593, U50,488 and their congeners spiradoline and enadoline, which have limited efficacy following oral administration (Béguin et al. 2008; Endoh et al. 1999). In a recent report, Prisinzano and Rothman (2008) suggested that analogs of the chemicals isolated from $S$. divinorum may prove to be excellent research tools and give greater insight into opioid receptor-mediated phenomena. Some synthetic analogs of salvinorin A with altered pharmacology have recently been reported (partial agonists and antagonists). However, these analogs show reduced binding affinity and retain the rapidly metabolized acetate which may be responsible for salvinorin A's brief duration of action. The pharmacological properties of salvinorin B MOM were recently characterized by Wang et al. (2008). This compound was reported to bind to KOP receptors with high selectivity and it displayed an approximately threefold higher affinity compared to U50,488 and salvinorin A. Salvinorin B MOM also acted as a full agonist at kappa receptors in functional assays, being approximately fivefold to sevenfold more potent than U50,488 and salvinorin A. All three of these kappa agonists internalized or downregulated kappa receptors to similar extents, and salvinorin B MOM displayed the greatest potency. In mice, salvinorin B MOM $(0.05-1.0 \mathrm{mg} / \mathrm{kg}$, s.c.) caused immediate and dose-dependent immobility lasting approximately $3 \mathrm{~h}$, which was blocked by the kappa antagonist, nor-BNI. Salvinorin B MOM $(0.5-5.0 \mathrm{mg} / \mathrm{kg}$, i.p.) also produced analgesia in the hot plate test and hypothermia in a dosedependent manner in rats. This compound was more potent than U50,488 in both tests and more efficacious than U50,488 in the hot plate test. These latter two in vivo effects were also blocked by nor-BNI, indicating kappa receptor-mediated actions. In contrast, salvinorin A $(10 \mathrm{mg} /$ $\mathrm{kg}$ ) elicited neither antinociception nor hypothermia $30 \mathrm{~min}$ after administration to rats. The findings reported by Wang et al. (2008) suggest that salvinorin B MOM is a potent and efficacious KOP receptor agonist with longer lasting in vivo effects than salvinorin $\mathrm{A}$. The current findings that salvinorin B MOM and salvinorin B EOM produce substitution in rats trained to discriminate U69,593 support previous reports that these agents are KOP agonists and that they are more potent than salvinorin A.

The recent upsurge in the recreational use and misuse of $S$. divinorum and the widespread availability of this plant and its fortified extracts require a comprehensive evaluation and characterization of the psychopharmacology and abuse liability of salvinorin A. There is also considerable evidence that alterations in KOP systems may underlie some of the neuroadaptive changes associated with compulsive drug seeking and relapse and the search for KOPs as possible pharmacotherapeutic agents has led to the recent development of synthetic analogs of salvinorin A with greater potency and a longer duration of action (Munro et al. 2008; Wang et al. 2008). Preclinical in vivo screening of these novel compounds is a crucial step in developing potential therapeutic agents for the treatment of substance abuse and dependence. A key component of these assessments includes screening these compounds for their subjective effects and abuse liability. Future studies aimed at further delineating the psychopharmacology of salvinorin A and related compounds offer an opportunity for the development of synthetic analogs of this novel naturally occurring KOP agonist for pharmacotherapeutic efficacy.

Acknowledgement The authors acknowledge Dr. Thomas Munro, Harvard Medical School, and McLean Hospital Corporation for their generous contribution of salvinorin A, salvinorin B EOM, and salvinorin B MOM.

\section{References}

Balster RL (1991) Drug abuse potential evaluation in animals. Br J Addict 86:1549-1558

Béguin C, Potter DN, DiNieri JA, Munro TA, Richards MR, Paine TA, Berry L, Zhao Z, Roth BL, Xu W, Liu-Chen L, Carlezon WA, Cohen BM (2008) N-methylacetamide analogue of Salvinorin A: a highly potent and selective kappa opioid receptor agonist with oral efficacy. J Pharmacol Exp Ther 324:188-195

Braida D, Limonta V, Pegorini S, Zani A, Guerini-Rocco C, Gori E, Sala M (2007) Hallucinatory and rewarding effect of salvinorin A in zebrafish: k-opioid and CB1-cannabinoid receptor involvement. Psychopharmacology 190:441-448

Braida D, Limonta V, Capurro V, Fadda P, Rubino T, Mascia P, Zani A, Gori E, Fratta W, Parolaro D, Sala M (2008) Involvement of kappa-opioid and endocannabinoid system on Salvinorin Ainduced reward. Biol Psychiatry 63:286-292

Butelman ER, Harris TJ, Kreek MJ (2004) The plant-derived hallucinogen, salvinoin A, produces kappa opioid agonist-like discriminative effects in rhesus monkeys. Psychopharmacology 127:220-224

Carlezon WA, Béguin C, DiNieri JA Bauman MH, Richards MR, Todtenkopf MS, Rothman RB, Ma Z, Lee DY, Cohen BM (2006) Depressive-like effects of the $\mathrm{K}$-opioid receptor agonist salvinorin 
A on behavior and neurochemistry in rats. J Pharmacol Exp Ther 316:440-447

Chavkin C, Sud S, Jin W, Stewart J, Zjawiony JK, Siebert DJ, Toth BA, Hufeisen SJ, Roth BL (2004) Salvinorin A, an active component of the hallucinogenic sage Salvia divinorum is a highly efficacious K-opioid receptor agonist: structural and functional considerations. J Pharmacol Exp Ther 308:1197-1203

Drug Enforcement Administration (2008) List of drugs and chemicals of concern. Available at http://www.deadiversion.usdoj. gov/drugs concern/salvia d/salvia d.htm. Accessed 1 October 2008

Endoh T, Matsuura H, Tajima A, Izumimoto N, Tajima C, Suzuki T, Saitoh A, Suzuki T, Narita M, Tseng L, Nagase H (1999) Potent antinociceptive effects of TRK-820, a novel kappa-opioid receptor agonist. Life Sci 65:1685-1694

Fantegrossi WE, Kugle KM, Valdes LJ 3rd, Koreeda M, Woods JH (2005) Kappa-opioid receptor-mediated effects of the plantderived hallucinogen, salvinorin $\mathrm{A}$, on inverted screen performance in the mouse. Behav Pharmacol 16:627-633

Gonzalez D, Riba J, Bouso JC, Gomez-Jabaro G, Barbanoj MJ (2006) Pattern of use and subjective effects of Salvia divinorum among recreational users. Drug Alcohol Depend 85:157-162

John TF, French LG, Erlichman JS (2006) The antinociceptive effect of salvinorin A in mice. Eur J Pharmacol 545:129-133

Li J, Rice KC, France CP (2008) Discriminative stimulus effects of 1(2,5-dimethoxy-4-methylphenyl)-2-aminopropane in rhesus monkeys. J Pharmacol Exp Ther 324:827-833

McCurdy CR, Sufka KJ, Smith GH, Warnick JE, Nieto MJ (2006) Antinociceptive profile of salvinorin A, a structurally unique kappa opioid receptor agonist. Pharmacol Biochem Behav 83:109-113

Munro TA, Duncan KK, Xu W, Wang Y, Liu-Chen LY, Carlezon WA, Cohen BM, Béguin C (2008) Standard protecting groups create potent and selective kappa opioids: salvinorin B alkoxymethyl ethers. Bioorg Med Chem 16:1279-1286

Ortega A, Blount JF, Manchand PS (1982) Salvinorin, a new transneoclerodane diterpene from Salvia divinorum (Labiatae). J Chem Soc 1:2505-2508

Prisinzano TE (2005a) Psychopharmacology of the hallucinogenic sage Salvia divinorum. Life Sci 78:527-531

Prisinzano TE $(2005 \mathrm{~b}) \mathrm{\kappa}$ Opioids as potential treatments for stimulant dependence. AAPS J 7:E593-E599 Available at http://www. aapsj.org

Prisinzano TE, Rothman RB (2008) Salvinorin A analogs as probes in opioid pharmacology. Chem Rev 108:1732-1743
Roth BL, Baner K, Westkaemper R, Siebert D, Rice KC, Steinberg S, Ernsberger P, Rothman RB (2002) Salvinorin A: a potent naturally occurring nonnitrogenous kappa opioid selective agonist. Proc Nat Acad Sci USA 99:11934-11939

Siebert DJ (1994) Salvia divinorum and salvinorin A: new pharmacologic findings. J Ethnopharmacol 43:53-56

Siebert DJ (2007) Legal status of Salvia divinorum. Available at http:// www.sagewisdom.org/legalstatus.html. Accessed 1 October 2008

Schuster CR, Johanson CE (1988) Relationship between the discriminative stimulus properties and subjective effects of drugs. Psychopharmacology 4:161-175

Sheffler DJ, Roth BL (2003) Salvinorin A: the "magic mint" hallucinogen finds a molecular target in the kappa opioid receptor. Trends Pharmacol Sci 24:107-109

Shippenberg TS, Chefer VI, Zapata A, Heidbreder CA (2001) Modulation of the behavioral and neurochemical effects of psychostimulants by K-opioid receptor systems. Ann N Y Acad Sci 937:50-73

Shippenberg TS, Zapata A, Chefer VI (2007) Dynorphin and the pathophysiology of drug addiction. Pharmacol Ther 116:306-321

Váldes LJ (1994) Salvia divinorum and the unique diterpene hallucinogen, salvinorin (divinorin) A. J Psychoactive Drugs $26: 277-283$

Váldes LJ, Butler WM, Hatfield GM, Paul AG, Koreeda M (1984) Divinorin A, a psychotropic terenoid, and divinorin B from the hallucinogenic Mexican mint, Salvia divinorum. J Org Chem 49:4716-4720

Váldes LJ, Diaz JL, Paul AG (1993) Ethnopharmacology of ska Maria Pastora (Salvia divinorum). J Ethnopharmacol 7:287-312

Wasson RG (1962) A new Mexican psychotropic drug from the mint family. Bot Mus Leaf Harv Univ 20:77-84

Wang Y, Chen Y, Xu W, Lee DY, Ma Z, Rawls SM, Cowan A, Liu-Chen LY (2008) 2-methoxymethyl-salvinorin B is a potent kappa opioid receptor agonist with longer-lasting action in vivo than salvinorin A. J Pharmacol Exp Ther 324:1073-1083

Willmore-Fordham CB, Krall DM, McCurdy CR, Kinder DH (2007) The hallucinogen derived from Salvia divinorum, salvinorin A, has k-opioid agonist discriminative stimulus effects in rats. Neuropharmacology 53:481-486

Yan F, Roth BL (2004) Salvinorin A: a novel and highly selective kappa-opioid receptor agonist. Life Sci 75:2615-2619

Zhang Y, Butelman ER, Schlussman SD, Ho A, Kreek MJ (2005) Effects of the plant-derived hallucinogen salvinorin A on basal dopamine levels in the caudate putamen and in a conditioned place aversion assay in mice agonist actions at kappa opioid receptors. Psychopharmacology 179:551-558 